\title{
A different disease, many diseases or mild asthma gone bad? Challenges of severe asthma
}

\author{
S.E. Wenzel
}

In the last 10 yrs, significant improvements have been made in the understanding of asthma. This improvement has partly been made through descriptive pathological and physiological studies, which have generally demonstrated a T-helper-2 pattern of local airway inflammation. Reducing this inflammation with corticosteroids (CS) leads to a better outcome for many asthmatics, with an impact on symptoms, quality of life and healthcare utilisation. Despite these improvements, it has become increasingly obvious that there remains a group of asthmatics who do not respond to treatment in the manner predicted by the response in milder asthmatics. The explanations for the inability to substantially impact outcomes in this population have ranged from psychological to pathological, but all have been based on limited data. In this issue of the European Respiratory Journal, the first report from a largescale, multicentre effort to understand severe asthma begins to offer some clues, while, however, raising many new questions [1].

The report from the European Network for Understanding Mechanisms of Severe Asthma (ENFUMOSA) suggests that severe asthma may, in fact, be a different disease to mildto-moderate, current-treatment responsive asthma [1]. This cross-sectional study evaluated over 150 severe asthmatics, who had been identified by their high corticosteroid use and healthcare utilisation, and who were well known to their physicians. These asthmatics differed from the control group of mild-to-moderate asthmatics in the predominance of females, aspirin sensitivity and lower level of atopy, either personally or by family history. As expected, physiologically the severe asthmatics were more obstructed, but, in addition, there was more air-trapping and, surprisingly, a slightly lower diffusing capacity than in control asthmatics. Sputum analysis suggested that despite high-dose inhaled and, in $30 \%$, oral steroids, eosinophil percentages remained at the level of controlled mild-to-moderate asthmatics (both still high, $\sim 10 \%$ ). Neutrophils were the only cell type significantly higher in severe asthmatics than mild-to-moderate asthmatics. As a result of these specific differences, the group suggested that, in their opinion, severe asthma was a "different" disease than the mild-to-moderate asthma that has been so extensively studied.

There is no doubt that severe asthma is different from mild asthma. The most obvious initial difference is the minimal efficacy of CS therapy, which is the main stay of asthma therapy for every other "persistent" asthma population. There are likely to be many reasons why a "disease" may be poorly $\mathrm{CS}$ responsive, not the least of which is the fact that it is a pathologically "different disease". However, the data from the ENFUMOSA study cannot exclude, in some patients, at least two other additional explanations: 1) classic CS resistance

Correspondence: S.E.Wenzel, National Jewish Medical and Research Center, 1400 Jackson St, Denver, CO 80206, USA. Fax: 1 3033981780. E-mail: wenzels@njc.org that prevents CS from functioning effectively on the same pathological process, as seen in milder asthma; or 2) that structurally altered (remodelled) airways prevent usual CS responses.

The higher level of aspirin sensitivity and lower level of atopy also suggest that severe asthma is a "different disease". There could be several explanations for these differences. Indeed, not everyone with severe asthma is aspirin sensitive and, although the incidence of atopy appears to be lower in the severe as compared to the control group, $60 \%$ of the severe asthma group demonstrated some level of atopy. As neither of these are "all-or-nothing" phenomena, it is more likely that that the range of responses indicates the heterogeneity of the severe asthma population. This would support the concept that several phenotypes may contribute to this severe asthma population. In an abstract presented at this year's American Thoracic Society meeting, information from the current author's database of severe asthma suggested that severe asthma with onset of disease after $18 \mathrm{yrs}$ of age is associated with a lower level of atopy, a higher degree of aspirin sensitivity, a greater eosinophilic presence and the suggestion of a more rapid decline in lung function than severe asthmatics whose disease began in childhood [2]. These asthmatics may represent the traditional classification of "intrinsic" asthma, which has been suggested in two other studies to have a more rapid decline in lung function [3]. However, even with this breakdown of adult- versus childhoodonset asthma, there are no biological/disease markers that clearly differentiate one severe asthma group from the other. In contrast, all of the severe asthmatics still have reversible airflow limitation and airway hyperresponsiveness. Overlap clearly exists and the current, very broad definition of asthma is also a limit.

The pathophysiological studies of the ENFUMOSA population present a group with a lower-than-expected diffusing capacity, greater airflow limitation and air trapping, and lower oxygen concentrations than control groups. These differences were seen in the light of similar eosinophil percentages in sputum and exhaled nitric oxide concentrations as in the control group. In contrast to the previous discussion, these data suggest that severe asthma could merely be an extension of mild asthma, a subgroup with an inadequate response to CS therapy. Using these parameters, there do not appear to be intrinsic differences between mild and severe asthma, just a spectrum of responses.

Finally, the severe asthmatics had higher neutrophil percentages in sputum (and peripheral blood). This increase has been shown in the majority of severe asthma studies but the reason for it is not clear [4-6]. CS are known to have "proneutrophilic" effects, and it would not be out of the question for the increase in neutrophils to be an effect of treatment, rather than a marker of the disease itself [7, 8]. Although neutrophil presence could suggest another disease, such as bronchiolitis obliterans, which can be almost impossible to 
differentiate from severe asthma radiologically and physiologically, further study would be required to confirm this assertion [9].

In 1999, the current author's group suggested that severe asthma was made up of at least two different diseases or phenotypes: one identified by the presence of eosinophils, despite high doses of CS, the other by their absence [5]. The ratio of the two groups over the years has been around 50:50. Pathologically, physiologically, and even clinically, these two groups remain quite distinct $[5,10]$. This distinction was especially noticeable when evaluating sub-basement membrane (SBM) thickness. The current author's group had previously published data indicating that, when evaluating the group as a whole, the SBM in severe asthma did not differ from that in milder asthmatics [11]. Yet, when evaluating individual data points, a clear suggestion of patterns emerged, with some individuals having a very thick SBM and others were remarkably thin SBM. It was not until the division of phenotypes (eosinophil presence or not) was applied that a group of severe asthma subjects that had a markedly thickened SBM emerged. At the same time, the group without eosinophils had an SBM thickness that only marginally differed from that of normal controls, suggesting that not only inflammatory processes but also type and degree of remodelling differentiated the groups. These structural changes may have then influenced various physiological and clinical parameters, including measures of airway collapsibility and near-fatal events. These data suggest that, rather than severe asthma being a different disease, it may consist of several (at least two) different diseases. Whether a similar approach to the data from the ENFUMOSA study will lead to similar conclusions is, of course, not known. However, this type of exploratory approach to this complex disease is encouraged.

While the explanations for the severe disease present in a minority of asthmatics remain poorly understood, it is clear that the increased awareness of the human and financial costs associated with this severe asthma population have promoted the development of several groups whose goal is to better understand this subpopulation. The ENFUMOSA group is moving forward into "Bio-Air", which will include endobronchial biopsy data. The National Institutes of Health have sponsored an eight-centre network to investigate, in a collective manner, the pathophysiology of the disease(s). Additionally, The Epidemiology and Natural History of Asthma: Outcomes and Treatment Regimens (TENOR) study is a 3-yr longitudinal study of difficult-to-treat asthma, evaluating risks, therapies and costs of disease in $>4,500$ subjects. This intensive and wide-ranging approach to severe asthma should produce a better understanding of the pathophysiological processes afflicting these patients.

Interestingly, none of these investigations have focused on psychiatric aspects of severe asthma, which, in the past, have been implicated as one of, if not the most, important aspect of the development of severe asthma [12, 13]. Psychiatric issues are clearly a part of any chronic illness and should not be dismissed. However, in many cases the psychiatric aspects may be the result rather than the cause of the disease, especially when standard therapy does not seem to be working. It is hoped for the sake of many, if not most, of these patients that the label of "crazy/noncompliant" will be removed, that there will be some recognition these individuals have a legitimate disease (or diseases) and that appropriate therapies will eventually be developed based upon these ongoing studies.

\section{References}

1. The ENFUMOSA Study Group. The ENFUMOSA crosssectional European multicentre study of the clinical phenotype of chronic severe asthma. Eur Respir J 2003; 22: 470477.

2. Miranda T, Strand MS, Trudeau JB, Fisher SJ, Wenzel SE. Severe asthmatics (SA) with late onset disease (LOD) have greater eosinophilic inflammation, airway obstruction and are less steroid responsive than early onset disease (EOD). Am J Respir Crit Care Med 2003; 167: A856.

3. Ulrik CS, Lange P. Decline of lung function in adults with bronchial asthma. Am J Respir Crit Care Med 1994; 150: 629-634.

4. Wenzel SE, Szefler SJ, Leung DYM, Sloan SI, Rex MD, Martin RJ. Bronchoscopic evaluation of severe asthma: persistent inflammation associated with high dose glucocorticoids. Am J Respir Crit Care Med 1997; 156: 737-743.

5. Wenzel SE, Schwartz LB, Langmack EL, et al. Evidence that severe asthma can be divided pathologically into two inflammatory subtypes with distinct physiologic and clinical characteristics. Am J Respir Crit Care Med 1999; 160: 1001-1008.

6. Louis R, Lau LCK, Bron AO, Roldaan AC, Radermecker M, Djukanovi R. The relationship between airways inflammation and asthma severity. Am J Respir Crit Care Med 2000; 161: 9-16.

7. Cox G. Glucocorticoid treatment inhibits apoptosis in human neutrophils. Separation of survival and activation outcomes. Am Assoc Immunologists 1995; 154: 4719-4725.

8. Schleimer RP, Freeland HS, Peters SP, Brown KE, Derse CP. An assessment of the effects of glucocorticoids on degranulation, chemotaxis, binding to vascular endothelium and formation of leukotriene B4 by purified human neutrophils. J Pharmacol Exp Ther 1989; 250: 598-605.

9. Jensen SP, Lynch DA, Brown KK, Wenzel SE, Newell JD. High-resolution CT features of severe asthma and bronchiolitis obliterans. Clin Radiology 2002; 57: 1078-1085.

10. Chu HW, Balzar S, Westcott JY, et al. Expression and activation of 15-lipoxygenase pathway in severe asthma: relationship to eosinophilic phenotype and collagen deposition. Clin Exp Allergy 2002; 32: 1558-1565.

11. Chu HW, Halliday JL, Martin RJ, Leung DYM, Szefler SJ, Wenzel SE. Collagen deposition in large airways does not appear to differentiate severe asthma from milder forms of the disease. Am J Respir Crit Care Med 1998; 158: 1936-1944.

12. Strunk RC, Mrazek DA, Fuhrmann GS, LaBrecque JF. Physiologic and psychological characteristics associated with deaths due to asthma in childhood. A case-controlled study. JAMA 1985; 254: 1193-1198.

13. ten Brinke A, Ouwerkerk ME, Zwinderman AH, Spinhoven $\mathrm{P}, \mathrm{Bel} \mathrm{EH}$. Psychopathology in patients with severe asthma is associated with increased health care utilization. Am J Respir Crit Care Med 2001; 163: 1093-1096. 